\title{
Composition of Coexisting Liquid Phases Determined by Rayleigh Interferometry
}

\author{
Alessandro Molisso • Onofrio Annunziata
}

Received: 19 April 2013/Accepted: 22 July 2013/Published online: 3 September 2013

(C) Springer Science+Business Media New York 2013

\begin{abstract}
Rayleigh interferometry is a precise macroscopic gradient technique that has been utilized for the determination of multicomponent diffusion coefficients. Because concentration gradients in multicomponent systems drive a diffusion-based partial separation of different solutes, this interferometric technique may be potentially used for the determination of solute concentrations. We have therefore theoretically examined how Rayleigh interferometry can be applied for the determination of composition of ternary aqueous mixtures. The effect of cross-term diffusion coefficients on the accuracy of this method is also discussed. Furthermore, since the poly(vinyl)alcohol+poly(ethylene)glycol+water system undergoes liquid-liquid phase separation (LLPS), we have experimentally characterized its LLPS boundary at $25{ }^{\circ} \mathrm{C}$. The corresponding tie-lines were characterized by determining the composition of the two coexisting liquid phases using Rayleigh interferometry.
\end{abstract}

Keywords Diffusion · Poly(vinyl alcohol) $\cdot$ Poly(ethylene glycol) $\cdot$ Liquid-liquid phase separation

\section{Introduction}

Rayleigh interferometry is a very precise technique for the determination of diffusion coefficients in multicomponent liquid mixtures [1,2]. This technique can give, at high precision, the refractive-index profile inside a cell in which the horizontal boundary between two solutions with different composition is vertically spreading by diffusion. The precision of the extracted refractive-index profile allows us to detect the presence of one,

\footnotetext{
A. Molisso · O. Annunziata ( $₫)$

Department of Chemistry, Texas Christian University, Fort Worth, TX 76129, USA

e-mail: o.annunziata@tcu.edu
}

\author{
A. Molisso \\ Department of Chemistry, "Federico II" University of Naples, 80126 Naples, Italy
}


two or even three solute diffusion modes [3]. This is the reason why this technique has been successful in the determination of multicomponent diffusion coefficients in aqueous systems [1-3].

The determination of the contribution of individual diffusion modes to the overall refractive-index profile may also find applications in quantitative chemical analysis. For example, the refractive-index profile associated with the diffusion of two known solutes can be used to determine their concentrations in solution. Experiments can be designed by preparing a boundary between the ternary solution of interest (diluted if needed) and the pure solvent. The goal of this paper is to explore how Rayleigh interferometry could be utilized to characterize the concentrations of two different nonionic polymers in ternary aqueous solutions. Specifically, we consider ternary mixtures of poly(vinyl alcohol) (PVA, $\left.\mathrm{MW}=100 \mathrm{~kg} \cdot \mathrm{mol}^{-1}\right)$ and poly (ethylene glycol) $\left(\mathrm{PEG}, \mathrm{MW}=8.0 \mathrm{~kg} \cdot \mathrm{mol}^{-1}\right)$ in water. The PEG+PVA+water system undergoes liquid-liquid phase separation due to the net repulsive interaction between these two polymers [4]. Thus, we utilize Rayleigh interferometry for determination of the PEG and PVA compositions of the two coexisting liquid phases (i.e., PEG/PVA partitioning).

These experiments are performed by employing Gosting's diffusiometer [5] operating in its Rayleigh interferometric mode [1]. This instrument, which is regarded as the world's most accurate optical interferometer for liquid-state diffusion coefficient measurements [1, 2, 5], was designed and assembled at the Institute for Enzyme Research in Madison, WI, during the 1960s and early 1970s under the direction of Louis J. Gosting [5]. It was later moved to Lawrence Livermore National Laboratory (1981-1991) under the supervision of Donald G. Miller [6], who also played a crucial role in the subsequent transfer to Texas Christian University, where it was operated under the supervision of John G. Albright [2] until his retirement in 2004. Miller and Albright with the technical help of Manfred E. Zeidler upgraded the Gosting's diffusiometer so that the acquisition of the Rayleigh interferometric pattern is automatized [7, 8]. This instrument is currently used in our research group [9].

\section{Materials and Methods}

\subsection{Materials}

Poly(ethylene glycol) with average molecular weight of $8.0 \mathrm{~kg} \cdot \mathrm{mol}^{-1}$, and PVA (99\% hydrolyzed, 85-124 $\mathrm{kg} \cdot \mathrm{mol}^{-1}$ ), were purchased from Sigma-Aldrich and used without further purification. Deionized water was passed through a four-stage Millipore filter system to provide high-purity water for all of the experiments. All solutions were prepared by weight using a Mettler-Toledo AT400 analytical balance starting from binary polymerwater stock solutions. PVA-water $(12 \% \mathrm{w} / \mathrm{w})$ and PEG-water $(30 \% \mathrm{w} / \mathrm{w})$ stock solutions were prepared in the following way. A solid polymer sample and water were mixed together in an Erlenmeyer flask immersed in a water bath at temperatures of about $95{ }^{\circ} \mathrm{C}$. The mixture was then extensively stirred for about $2 \mathrm{~h}$. Stirring was continued at room temperature for at least other $6 \mathrm{~h}$.

\subsection{Liquid-Liquid Phase Boundary}

The liquid-liquid phase boundary of the PVA+PEG+water system was determined at $25^{\circ} \mathrm{C}$. Known amounts of PEG+water and PVA+water stock solutions and water were stirred together in a $15 \mathrm{~mL}$ test tube so that a biphasic system was obtained at $25^{\circ} \mathrm{C}$. The 
cloudy sample was placed in a water bath at $25.00 \pm 0.01{ }^{\circ} \mathrm{C}$. Small and known amounts of water were then incrementally added to the cloudy samples until opacification was observed to disappear in the thermostatted sample. All samples were scrutinized by using a magnification lens. The polymer weight fractions on the phase boundary were taken to be the average between those of the first clear sample and those of the last opaque sample.

\subsection{Determination of Tie-Lines}

Known amounts of PEG+water and PVA+water stock solutions and water were mixed together in a $15 \mathrm{~mL}$ test tube so that a biphasic system was obtained at $25^{\circ} \mathrm{C}$. Biphasic samples were first extensively stirred at room temperature. These samples were then placed in the water bath at $25.00 \pm 0.01{ }^{\circ} \mathrm{C}$ and left for $24 \mathrm{~h}$ to ensure macroscopic phase separation by gravity. The top (I) and bottom (II) coexisting phases were then separated from each other and individually placed in the water bath for about $6 \mathrm{~h}$. Individual phases were then centrifuged so that residual amounts (if present) of their complementary phase could be removed. Individual phases were then diluted with water so that their final total polymer weight percent in water was lower than $1 \%$.

The mass concentrations of PVA(1) and PEG(2), $C_{1}$ and $C_{2}$, of a dilute ternary solution was then determined using the Gosting diffusiometer. Mass concentrations (in $\mathrm{g} \cdot \mathrm{dm}^{-3}$ ) were then converted into weight percent, $w_{1}$ and $w_{2}$, by measuring the solution densities. All density measurements were made with a Mettler-Paar DMA40 density meter, thermostatted with water from a large, well-regulated $\left( \pm 0.001{ }^{\circ} \mathrm{C}\right)$ water bath. These concentrations were then used (after dilution correction) to calculate the weight percents, $\left(w_{1}^{(\mathrm{I})}, w_{2}^{(\mathrm{I})}\right)$ and $\left(w_{1}^{(\mathrm{II})}, w_{2}^{(\mathrm{II})}\right)$, of the two coexisting liquid phases I (bottom) and II (top), respectively.

\subsection{Gosting Diffusiometer}

Rayleigh interferometry provides a one-dimensional profile of the refractive index inside a vertical channel filled with liquid. Diffusion inside the channel is brought about by formation of an initial sharp boundary between the bottom solution and the top solution with different compositions and, consequently, different refractive indices. A comprehensive description of the Gosting diffusiometer can be found in [2] and references cited therein. In brief, a typical diffusion experiment starts from preparing a sharp boundary (using a peristaltic pump) between two uniform bottom and top solutions. In our case, the bottom and top solutions are represented by the diluted polymer aqueous phase and pure water, respectively. These two solutions are located inside a vertical channel with inside width of $2.5057 \mathrm{~cm}$. The light source used for generating the Rayleigh interference pattern is a He$\mathrm{Ne}$ Uniphase laser with wavelength $\lambda=543.5 \mathrm{~nm}$. A cell holder is located inside the water bath. The temperature of the bath at $25.00{ }^{\circ} \mathrm{C}$ was regulated with a precision of $\pm 0.001{ }^{\circ} \mathrm{C}$. The cell holder supported a Tiselius cell, where diffusion occurs, and a mask, which consists of a double slit. Here, the laser beam is split into two parts: one going through the diffusion channel of the Tiselius cell and the other passing through the water bath (reference channel). A pair of two cylinder lenses focuses the diffusion channel onto the detector, where the Rayleigh interference pattern is observed and recorded. Rayleigh fringes shift horizontally as the refractive index inside the diffusion channel changes with vertical height. This gives direct information about the total number of fringes, $J$, and thus the normalized refractive index profile. To minimize effects due to the concentration dependence of diffusion coefficients and refractive-index increments, Creeth's 
antisymmetrization procedure (Creeth-pair method) [10] was applied. To minimize effects of polymer polydispersity, diffusion measurements on binary polymer-water systems were used to remove the polydispersity contribution from the refractive-index profile of the ternary systems as described in [11]. The method of non-linear least squares [12] was then applied to the corrected refractive-index profile in order to extract all needed diffusion parameters.

\section{Theory}

In this section, we outline the method to extract the solute mass concentrations, $C_{1}$ and $C_{2}$, of a ternary solution. The normalized refractive-index profile inside the channel is described by the function $f=2(n-\bar{n}) / \Delta n$ where $n(x)$ is the refractive index at a given position, $x$, inside the channel, and $\bar{n}$ and $\Delta n$ are, respectively, the average and the difference in refractive indices between the two solutions. The normalized refractive index is obtained by locating the position of the fringe of the resulting Rayleigh interference pattern. One important parameter extracted from a given diffusion measurement is the total number of interference fringes, $J$ [1]. If the top solution is pure water, then we have:

$$
J=R_{1} C_{1}+R_{2} C_{2}
$$

where $R_{i}$ is an instrumental constant directly proportional to the refractive-index contribution of solute $i$. For a system with three components (two solutes and one solvent), the generalized Fick's first law [2] is given by

$$
\begin{aligned}
& -J_{1}=D_{11} \nabla C_{1}+D_{12} \nabla C_{2} \\
& -J_{2}=D_{21} \nabla C_{1}+D_{22} \nabla C_{2}
\end{aligned}
$$

In Eqs. $2 \mathrm{a}$ and $2 \mathrm{~b}, J_{i}$ is the mass flux of solute $i, C_{j}$ is the mass concentration of solute $j$, and $D_{i j}$ is the diffusion coefficient that relates the flux of solute $i$ to the concentration gradient of $j$. Main-term diffusion coefficients, $D_{11}$ and $D_{22}$, describe the flux of each solute due to its own concentration gradient, while the cross-term diffusion coefficients, $D_{12}$ and $D_{21}$, are responsible for the flux of a solute due to the concentration gradient of the other solute. The cross-term $D_{i j}$ approaches zero as $C_{i}$ approaches zero at constant $C_{j}$. Thus cross-term diffusion coefficients may be negligible in solutions dilute in that solute.

The normalized refractive-index profile associated with the free-diffusion boundary condition is mathematically described by the linear combination of two error functions [1]:

$$
f=\Gamma_{1} \operatorname{erf}\left(y / \sqrt{D_{1}}\right)+\Gamma_{2} \operatorname{erf}\left(y / \sqrt{D_{2}}\right)
$$

where $y \equiv x / 2 \sqrt{t}, t$ is time, and $D_{i}$ (with $i=1,2$ ) are the two eigenvalues of the $D_{i j}$ matrix characterizing the two diffusion modes. If we define our solute components such that $D_{11}<D_{22}$, we can write:

$$
\begin{aligned}
& D_{1}=\frac{1}{2}\left(T-\sqrt{T^{2}-4 \Delta}\right)=D_{11}-\frac{1}{4} \frac{D_{12} D_{21}}{D_{11}-D_{22}}+\ldots \\
& D_{2}=\frac{1}{2}\left(T+\sqrt{T^{2}-4 \Delta}\right)=D_{22}+\frac{1}{4} \frac{D_{12} D_{21}}{D_{11}-D_{22}}+\ldots
\end{aligned}
$$

where $T \equiv D_{11}+D_{22}, \Delta \equiv D_{11} D_{22}-D_{12} D_{21}$, and the right-hand-side of Eqs. 4a and $4 \mathrm{~b}$ represents Taylor's series expansions to first order with respect to $D_{12} D_{21} /\left(D_{11}-D_{22}\right)$. In 
the presence of net solute-solute repulsive interactions, the cross-term diffusion coefficients are expected to be both positive [13-15] and $D_{12} D_{21}>0$. This implies that $D_{1}<D_{11}$ and $D_{2}>D_{22}$. If the concentration of one of the two solutes is low, then the magnitude of one of the two cross-term diffusion coefficient is small. In this case, we have $D_{1} \approx D_{11}$ and $D_{2} \approx D_{22}$ according to Eqs. $4 \mathrm{a}$ and $4 \mathrm{~b}$. The constants $\Gamma_{i}$ in Eq. 3, which satisfy the normalization condition $\Gamma_{1}+\Gamma_{2}=1$, characterize the relative weights of the two diffusion modes to the overall refractive-index profile. The expressions of $\Gamma_{i}$ can be taken from the original work of Fujita and Gosting [16]. These expression are rewritten in the following way:

$$
\Gamma_{i}=\frac{\left[D_{j}-D_{i i}-\left(R_{j} / R_{i}\right) D_{j i}\right] \alpha_{i}+\left[D_{j}-D_{j j}-\left(R_{i} / R_{j}\right) D_{i j}\right] \alpha_{j}}{D_{j}-D_{i}} \text { with } i, j=1,2 \text { and } i \neq j
$$

where $\alpha_{i} \equiv R_{i} C_{i} / J$ and $\alpha_{1}+\alpha_{2}=1$. The key approximation that allows us to determine $C_{i}$ from $\Gamma_{i}$ is based on the limiting condition: $\Gamma_{i}=\alpha_{i}$ applying when $D_{12}=D_{21}=0$. This limiting equality is expected to be a reasonable approximation in dilute solutions of nonionic solute components. Our approach for the determination of the values of $C_{1}$ and $C_{2}$ is based on the application of the method of the non-linear least squares as developed by Miller [12] to Eq. 3. The values of $\Gamma_{i}$ can be converted into the corresponding $C_{i}$ using:

$$
C_{i}=J \frac{\Gamma_{i}}{R_{i}} \text { with } i=1,2
$$

Diffusion measurements on binary polymer-water systems with known solute concentrations can be used to extract the needed values of $R_{i}$ for the application of Eq. 6 . These values can be assumed to be the same as those in dilute ternary systems. Indeed previous ternary-diffusion measurements on polymer systems show that this assumption is acceptable even for concentrated mixtures [11].

\section{Results and Discussion}

The total polymer concentration in the investigated coexisting liquid phases is of the order $\approx 100 \mathrm{~g} \cdot \mathrm{dm}^{-3}$. Samples were diluted so that the total polymer concentration was reduced to $\approx 5 \mathrm{~g} \cdot \mathrm{dm}^{-3}$. Diffusion measurements on binary PVA(1)+water and PEG(2)+water systems were performed at similar concentrations at $25^{\circ} \mathrm{C}$. Binary polymer concentrations $C_{i}$, binary diffusion coefficients $D_{i}$, and the corresponding $R_{i}$ values are reported in Table 1.

In Table 2, we report our experimental compositions for the liquid-liquid phase boundary. These data, which are shown in Fig. 1 (open circles), were used to identify compositions $\left(w_{1}^{0}, w_{2}^{0}\right)$ that could approximately generate equal amounts of the two coexisting liquid phases (I) and (II) according to lever rule. The composition of the prepared samples (A, B and C) are reported in Table 3 (see also labeled open squares in

Table 1 Diffusion results for binary polymer+water systems at $25^{\circ} \mathrm{C}$

\begin{tabular}{llll}
\hline & $C_{i}\left(\mathrm{~g} \cdot \mathrm{dm}^{-3}\right)$ & $D_{i}\left(10^{-9} \mathrm{~m}^{2} \cdot \mathrm{s}^{-1}\right)$ & $R_{i}\left(\mathrm{~g} \cdot \mathrm{dm}^{-3}\right)$ \\
\hline PVA (1) & 5.208 & 0.02663 & 7.216 \\
PEG (2) & 4.608 & 0.09002 & 6.209 \\
\hline
\end{tabular}


Fig. 1). Our diffusion results on diluted phases are also reported in Table 3. Due to net PVA-PEG repulsive interactions, phases (I) and (II) are enriched in PVA and PEG, respectively. This implies that one solute component dominates in individual solutions. As a consequence of this phenomenon, the application of the method of non-linear least squares to Eq. 3 yielded unrealistic values of the $D_{j}$ associated with the minor solute component. This is related to the fact that the corresponding $\Gamma_{j}$ value was found to be relatively small. Hence, the value of $D_{j}$ was set to be constant and equal to the corresponding binary value $D_{i}$ in Table 1 .

Table 2 Compositions of the liquid-liquid phase boundary

\begin{tabular}{lrcc}
\hline$w_{2}(\%)$ & $w_{1}(\%)$ & $w_{2}(\%)$ & $w_{1}(\%)$ \\
\hline 0.73 & 11.89 & 2.90 & 1.99 \\
1.33 & 7.89 & 3.02 & 1.46 \\
1.54 & 6.96 & 3.69 & 0.74 \\
1.77 & 5.38 & 4.44 & 0.45 \\
2.08 & 4.71 & 5.82 & 0.23 \\
2.12 & 4.14 & 7.66 & 0.06 \\
2.33 & 3.17 & 9.66 & 0.03 \\
2.43 & 3.37 & 10.51 & 0.02 \\
\hline
\end{tabular}

Fig. 1 Phase diagram of the PVA(1)+PEG(2)+water system showing the liquid-liquid phase boundary (open circles) at $25^{\circ} \mathrm{C}$. The solid curve is a guide for the eye. The pair of points $\left(w_{1}^{(\mathrm{I})}, w_{2}^{(\mathrm{I})}\right)$ and $\left(w_{1}^{(\mathrm{II})}, w_{2}^{(\mathrm{II})}\right)$, representing the coexisting liquid phases (I) and (II) (closed squares), are connected by tie lines (solid lines). The overall compositions (open squares) of the samples, $\left(w_{1}^{0}, w_{2}^{0}\right)$, prepared to generate the coexisting phases, are also included and labeled as shown in Table 1. The critical point (closed diamonds) composition $\left(w_{1}^{\mathrm{c}}, w_{2}^{\mathrm{c}}\right)$ was estimated by a linear extrapolation of $\left(w_{i}^{(\mathrm{I})}+\right.$ $\left.w_{i}^{(\mathrm{II})}\right) / 2$ (with $i=1,2$ ) to $\left|w_{i}^{(\mathrm{II})}-w_{i}^{(\mathrm{I})}\right|=0$

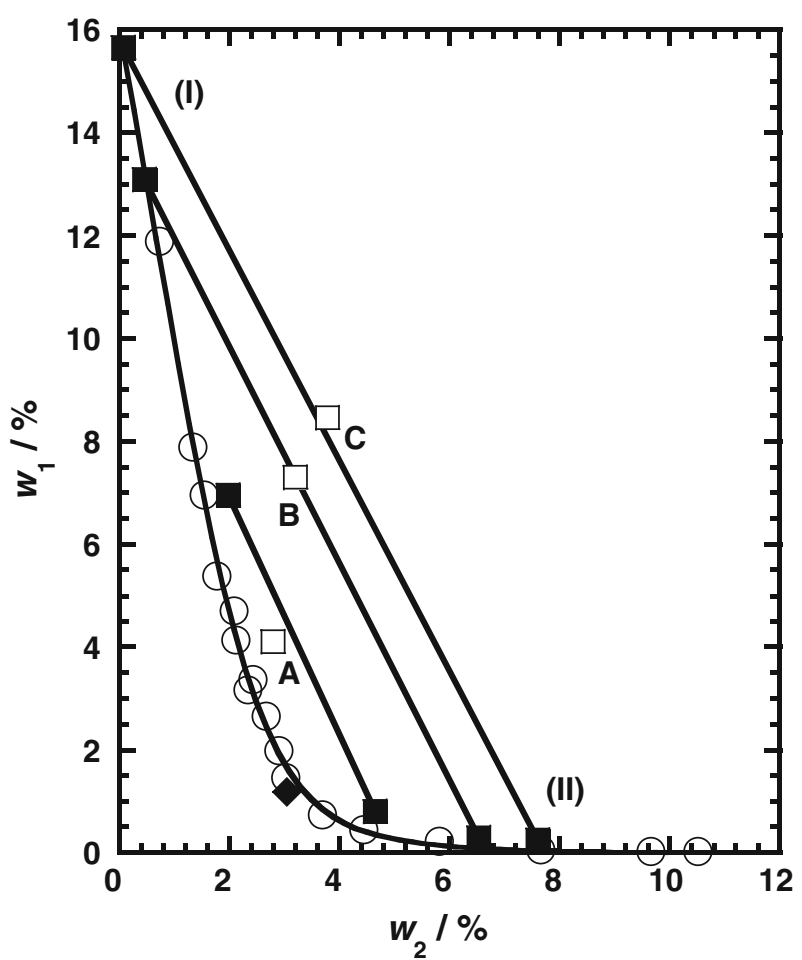




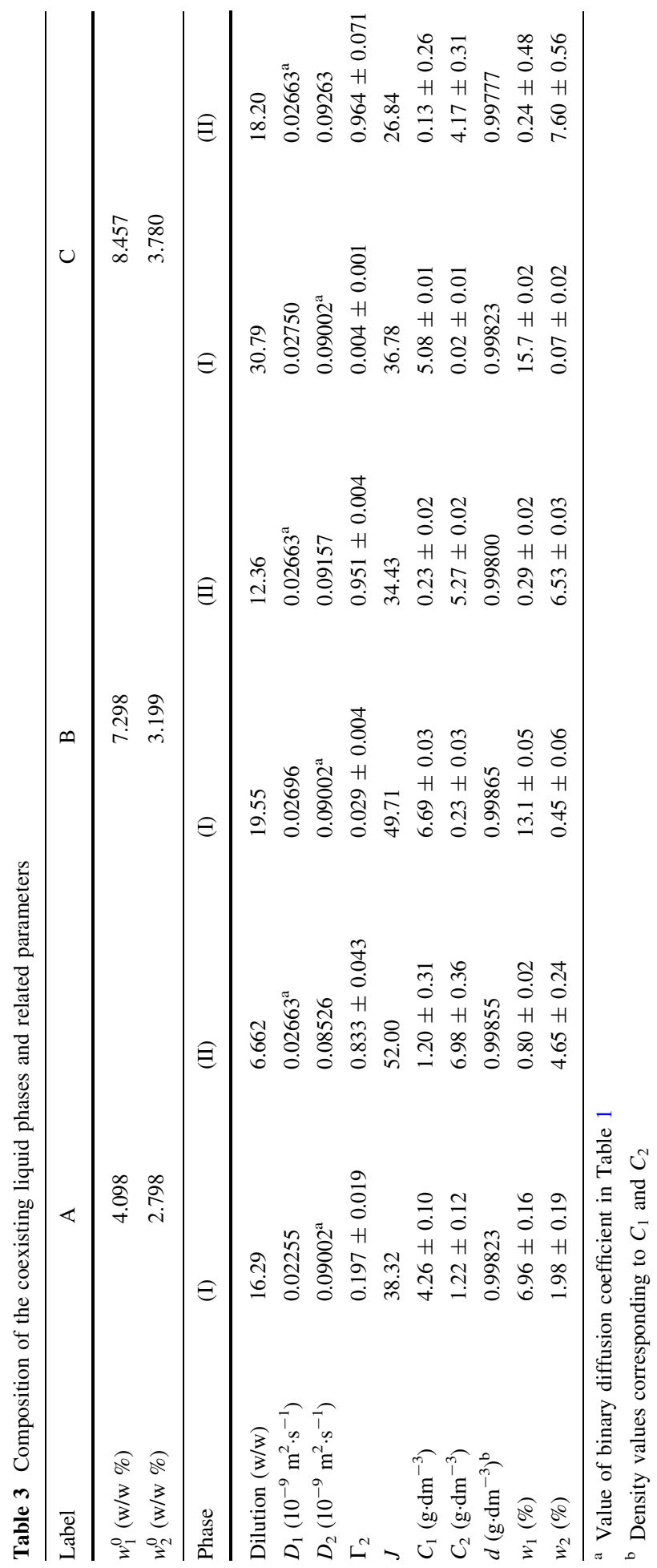


The compositions of the determined coexisting liquid phases are shown in Fig. 1. As we can see, very good agreement was obtained for samples B and C. However, the pair of compositions related to sample A was found to be slightly inside the phase boundary. Furthermore the initial compositions $\left(w_{1}^{0}, w_{2}^{0}\right)$ are expected to be located on the tie lines connecting compositions of coexisting phases $\left(w_{1}^{(\mathrm{I})}, w_{2}^{(\mathrm{I})}\right)$ and $\left(w_{1}^{(\mathrm{II})}, w_{2}^{(\mathrm{II})}\right)$. While very good agreement was obtained for samples B and C, the composition of sample A was found to be slightly below the corresponding tie line. Repeated measurements on sample A lead to the same results within the experimental error.

One possible reason for the discrepancy observed for sample A may be attributed to non-negligible cross-diffusion effects in Eq. 5. We now examine the role of cross-term diffusion coefficients on the deviation of $\Gamma_{i}$ from $\alpha_{i}$. If we include only terms to first order in $D_{i j} /\left(D_{j j}-D_{i i}\right)$, then Eq. 5 becomes:

$$
\Gamma_{i}=\alpha_{i}\left(1-\frac{\left(R_{j} / R_{i}\right)\left(D_{j i} / C_{j}\right)+D_{i i}\left(D_{i j} / C_{i}\right)}{D_{j j}-D_{i i}} C_{j}\right)+\ldots
$$

Note that cross-term diffusion coefficients in Eq. 7 were rewritten as quotients, $D_{i j} / C_{i}$, since $D_{i j}$ is directly proportional to $C_{i}$ to first order. According to Eq. 7, the value of $\Gamma_{i}$ overestimates (underestimates) the value of $\alpha_{i}$ for the relatively fast (slow) diffusing solute when both cross-term diffusion coefficients are positive. Thus, we conclude that $\Gamma_{2}>\alpha_{2}$ (and $\Gamma_{1}<\alpha_{1}$ ) in our case. Furthermore, it is also important to observe that the condition $\Gamma_{i}=\alpha_{i}$ is respected when $C_{j} \rightarrow 0$ (see Eq. 7). This limiting condition is analogous to Raoult's law describing the liquid-vapor equilibrium of real mixtures.

This analysis of Eq. 7 is consistent with our experimental results. For samples B and C, the concentration of one of the two solute components is very small (with a mass contribution being less than $5 \%$ of that of the other solute). This implies that $\Gamma_{i}$ is expected to yield relatively accurate values of concentrations for the dominant polymer. On the other hand, the concentrations of the minor solute component in sample A are relatively high. Thus, according to Eq. 7, this discrepancy might be related to $\Gamma_{2}$ in Table 3 being appreciably larger than $\alpha_{2}$. For example, we have observed that better agreements with both the liquid-liquid phase boundary and $\left(w_{1}^{0}, w_{2}^{0}\right)$ are obtained if the value of $\Gamma_{2} \approx 0.20$ for phase (I) of sample A corresponds to the lower value of $\alpha_{2} \approx 0.15$.

\section{Conclusions}

The application of diffusion-based partial separation of solute components to the determination of the composition of coexisting liquid phases in ternary polymer aqueous systems using Rayleigh interferometry was described. Satisfactory accuracy was found when one of the two polymer components dominates. Systematic studies of multicomponent diffusion on ternary solutions with known compositions should be performed to assess precision and accuracy of this method in quantitative chemical analysis.

Acknowledgments The authors are in debt with John G. Albright and Donald G. Miller for their invaluable assistance with the Gosting diffusiometer throughout these past years. Alessandro Molisso thanks Ornella Ortona, Roberto Sartorio and Luigi Paduano for their support and guidance. This work was supported by the TCU Research and Creative Activity Funds. 


\section{References}

1. Miller, D.G., Albright, J.G.: Optical methods. In: Wakeham, W.A., Nagashima, A., Sengers, J.V. (eds.) Measurement of the transport properties of fluids: experimental thermodynamics, vol. III, pp. 272-294. Blackwell Scientific Publications, Oxford (1991)

2. Albright, J.G., Annunziata, O., Miller, D.G., Paduano, L., Pearlstein, A.J.: Precision measurements of binary and multicomponent diffusion coefficients in protein solutions relevant to crystal growth: lysozyme chloride in water and aqueous $\mathrm{NaCl}$ at $\mathrm{pH} 4.5$ and $25{ }^{\circ} \mathrm{C}$. J. Am. Chem. Soc 121, 3256-3266 (1999)

3. Annunziata, O., Vergara, A., Paduano, L., Sartorio, R., Miller, D.G., Albright, J.G.: Quaternary diffusion coefficients in a protein-polymer-salt-water system determined by Rayleigh interferometry. J. Phys. Chem. B 113, 13446-13453 (2009)

4. Pereira, M., Wu, Y.-T., Madeira, P., Venancio, A., Macedo, E., Teixeira, J.: Liquid-liquid equilibrium phase diagrams of new aqueous two-phase systems: ucon 50-HB5100+ammonium sulfate+water, ucon 50-HB5100+poly(vinyl alcohol)+water, ucon 50-HB5100+hydroxypropyl starch+water, and poly(ethylene glycol) 8000+poly(vinyl alcohol)+water. J. Chem. Eng. Data 49, 43-47 (2004)

5. Gosting, L.J., Kim, H., Loewenstein, M.A., Reinfelds, G., Revzin, A.: A versatile optical diffusiometer including a large optical bench of new design. Rev. Sci. Instrum 44, 1602-1609 (1973)

6. Mathew, R., Paduano, L., Albright, J.G., Miller, D.G., Rard, J.A.: Isothermal diffusion coefficients for $\mathrm{NaCl}-\mathrm{MgCl}_{2}-\mathrm{H}_{2} \mathrm{O}$ at $25^{\circ} \mathrm{C}$. 2. Low concentrations of sodium $\mathrm{NaCl}$ with a wide range of $\mathrm{MgCl}_{2}$ concentrations. J. Phys. Chem 93, 4366-4370 (1989)

7. Miller, D.G., Albright, J.G., Mathew, R., Lee, C.M., Rard, J.A., Eppstein, L.B.: Isothermal diffusion coefficients of $\mathrm{NaCl}-\mathrm{MgCl}_{2}-\mathrm{H}_{2} \mathrm{O}$ at $25^{\circ} \mathrm{C}$. 5. Solute concentration ratio of 1:1 and some Rayleigh results. J. Phys. Chem 97, 3885-3899 (1993)

8. Rard, J.A., Albright, J.G., Miller, D.G., Zeidler, M.E.: Ternary mutual diffusion coefficients and densities of the system $\left\{\mathrm{z}_{1} \mathrm{NaCl}+\left(1-z_{1}\right) \mathrm{Na}_{2} \mathrm{SO}_{4}\right\}(\mathrm{aq})$ at $298.15 \mathrm{~K}$ and a total molarity of $0.5000 \mathrm{~mol} \mathrm{dm}^{-3}$. J. Chem. Soc. Faraday Trans 92, 4187-4197 (1996)

9. Zhang, H., Annunziata, O.: Macromolecular hydration compared with preferential hydration and their role on macromolecule-osmolyte coupled diffusion. Phys. Chem. Chem. Phys 11, 8923-8932 (2009)

10. Creeth, J.M., Gosting, L.J.: Studies of free diffusion in liquids with the Rayleigh method. II. An analysis for systems containing two solutes. J. Phys. Chem 62, 58-65 (1958)

11. Zhang, H., Annunziata, O.: Effect of macromolecular polydispersity on diffusion coefficients measured by Rayleigh interferometry. J. Phys. Chem. B 112, 3633-3643 (2008)

12. Miller, D.G.: A method for obtaining multicomponent diffusion coefficients directly from Rayleigh and Gouy fringe position data. J. Phys. Chem 92, 4222-4226 (1988)

13. Vitagliano, V., Sartorio, R.: Diffusion in ternary systems. J. Phys. Chem 74, 2949-2956 (1970)

14. Vergara, A., Paduano, L., Sartorio, R.: Multicomponent diffusion in systems containing molecules of different size. 4. Mutual diffusion in the ternary system tetra(ethylene glycol)-di(ethylene glycol)water. J. Phys. Chem. B 105, 328-334 (2001)

15. Annunziata, O.: On the role of solute solvation and excluded-volume interactions in coupled diffusion. J. Phys. Chem. B 112, 11968-11975 (2008)

16. Fujita, H., Gosting, L.J.: An exact solution of the equations for free diffusion in three-component systems with interacting flows, and its use in evaluation of the diffusion coefficients. J. Am. Chem. Soc 78, 1099-1106 (1956) 